\title{
Antioxidant fractions and phenolic constituents from leaves of Pluchea carolinensis and Pluchea rosea
}

\author{
Wilmer Hervet Perera Cordova ${ }^{1}$, Jean-Noël Wauters ${ }^{2}$, Claire Kevers ${ }^{1}$, Michel Frédérich ${ }^{2}$, Jacques Dommes \\ ${ }^{I}$ Plant Molecular Biology and Biotechnology Unit, Institute of Botany B22, University of Liège, Sart Tilman, BE-4000 Liège, Belgium \\ ${ }^{2}$ Laboratory of Pharmacognosy, Institute of Pharmacy B36, University of Liège, Sart Tilman, BE-4000 Liège, Belgium
}

\begin{abstract}
Objective: The objective is to evaluate the antioxidant potential of several polar fractions of Pluchea carolinensis and Pluchea rosea as well as pure chemicals, some of them quantified in both species by highperformance liquid chromatography (HPLC). Methods: The antioxidant potential of polar fractions and pure chemicals were assayed by 2,2-diphenyl-1-picrylhydrazyl (DPPH) and oxygen radical potential methods. The phenolic content was performed by using Folin-Ciocalteu's reagent. Specific phenolic acids and flavonoids were quantified by diode array detector-reversed phase-HPLC. Results: The highest DPPH antioxidant potential expressed in milligrams of trolox equivalents per gram of dry extract (mg TE/gDE) were frequently measured in fractions from $n$-butyl alcohol, i.e., 2 (192.1 \pm 0.3$)$; 6 (181.0 \pm 0.1$)$ of $P$. carolinensis and in fraction 7 (188.1 \pm $5.5)$ of $P$. rosea while for oxygen radical scavenging capacity (mg TE/gDE) assay fraction $2(543.0 \pm 64.6)$ and 4 $(501.4 \pm 49.7)$ of $P$. carolinensis and $3(401.3 \pm 16.1)$ and $6(401.3 \pm 16.1)$ of $P$. rosea showed the best results. Some flavonoids and phenolic acids were also assayed; all of them showed highest oxygen radical absorbance capacity values. Conclusion: We report the antioxidant potential of polar fractions, as well as of some pure phenolics responsible of the antioxidant potential. Some phenolics were identified and quantified for the first time in both species. Apparently, caffeoylquinic acid derivatives contribute more significant to the total antioxidant potential of the extracts.
\end{abstract}

Keywords : Antioxidant potential ; caffeoylquinic acid derivatives ; cinnamic acids ; flavonoids ; phenolic profile

\section{INTRODUCTION}

The genus Pluchea belongs to one of the most diverse botanical family, Asteraceae. Pluchea counts about 80 species of small herbs and shrubs ${ }^{1}$ and a large number of these taxa (30-40) thrive in tropical regions. In Cuba, three species are reported: Pluchea carolinensis (Jacq.) G. Don., Pluchea odorata (L.) Cass, and Pluchea rosea Godfrey.

Pluchea is frequently identified as a source of antioxidants, ${ }^{2}$ of antimicrobial compounds,${ }^{3}$ of fungicides, ${ }^{4}$ of insecticides, ${ }^{5}$ of anti-inflammatory chemicals ${ }^{6}$ and of allelopathic compounds ${ }^{7}$ among others pharmacological properties. The antioxidant potential of some plants has been described in literatures. ${ }^{8,9}$ Cuban species of Pluchea has recently been evaluated. In these species, high antioxidant capacity has been correlated with the content of phenolic compounds. ${ }^{10}$ Hence, the Cuban Pluchea species are considered as an interesting source of chemicals for preparing functional foods.

Many of the Pluchea species are known as aromatic plants with a characteristic scent produced by complex mixtures of volatile terpenoids. Studies based on the identification or isolation of secondary metabolites suggested that terpenoids are the most widespread metabolites in the genus. ${ }^{11,12}$ Eudesmane derivatives are a sesquiterpene group widely distributed in the genus. ${ }^{13,14}$ Phenolic compounds are the second most widespread metabolites in Pluchea. ${ }^{15}$ Several flavonols mainly of the quercetin, kempferol and quercetagetin types have been identified. ${ }^{15-17}$ In addition, a few flavonol aglycones have been previously isolated from $P$. carolinensis,${ }^{18}$ no report from $P$. rosea and various flavonoids have been identified from $P$. odorata. ${ }^{16}$ In addition, polyphenols including flavonoids have been reported to exhibit a wide range of biological activities and their effects are mainly attributed to the antioxidant properties. ${ }^{19}$ 
As far as we know, the chemical composition of phenolic compounds of the Cuban Pluchea species and thus, the phytochemicals responsible of the antioxidant capacity of the crude extracts are still unknown. Hence, the present study aim to determine the major phenolic phytochemicals in the ethyl acetate (EtOAc) and $n$-butanol ( $n$ $\mathrm{BuOH}$ ) extracts of $P$. carolinensis and $P$. rosea. The antioxidant potential of several fractions was evaluated by a phenolic screening by high-performance liquid chromatography (HPLC). Furthermore, the antioxidant potential of various phenolic identified and other analogous compounds was assayed using 2,2-diphenyl-1 picrylhydrazyl (DPPH) and oxygen radical scavenging capacity (ORAC) assay.

\section{MATERIALS AND METHODS}

\section{Plant material}

P. carolinensis (voucher HAC 41725) was collected in Sierra del Rosario, Pinar del Río, Cuba (Longitude: $82^{\circ}$ 56' 57" and Latitude: $22^{\circ} 50^{\prime}$ 56") in March 2008 during their flowering stage and $P$. rosea (voucher LS 16648) in Ciénaga de Zapata, Matanzas (Longitude: $87^{\circ} 10^{\prime} 47^{\prime \prime}$ and Latitude: $22^{\circ} 22^{\prime} 89^{\prime \prime}$ ) in 2006. All the vouchers were authenticated by MSc. Ramona Oviedo Prieto and Dr. Pedro Herrera Oliver, and deposited at the HAC herbarium of the "Instituto de Ecologia y Sistemática."

\section{Chemicals}

The flavonoids standards: Quercetin, kempferol, myricetin, isorhamnetin, quercetagetin, quercitrin, casticin, herbacetin, hyperoside, spiraeoside, apigenin, luteolin, daidzein, daidzin, genistein, genistin, ononin, rutin, naringenin, taxifolin, quercetin-3-O-glucopyranoside, kaempferol-3-O-rutinoside were purchased from Extra synthèse (France). The phenolic acids: Ferulic acid, ellagic acid, rosmarinic acid, vanillic acid, and p-hydroxy benzoic acid were purchased from Aldrich, as well as the reagent 2',2-azobis(2-amidinopropane) dihydrochloride. Gallic acid, chlorogenic acid, syringic acid, p-coumaric acid, o-coumaric acid, sinapic acid, gentisic acid, caffeic acid, salicylic acid were purchased from Sigma, as well as DPPH and Trolox. FolinCiocalteu reagent was purchased from $\mathrm{BDH}$.

\section{Preparation of the extracts and fractionation}

A total of $1293 \mathrm{~g}$ and $895 \mathrm{~g}$ of dried, powdered leaves of $P$. carolinensis and P. rosea respectively, were extracted using ethanol: $\mathrm{H}_{2} \mathrm{O}(7: 3 \mathrm{v} / \mathrm{v})$ macerations. Ethanol was evaporated under reduced pressure. The resulting aqueous solutions were successively fractioned with solvent of increasing polarity: $n$-hexane, chloroform $\left(\mathrm{CHCl}_{3}\right)$, EtOAc and $n$-BuOH. ${ }^{10}$ Five grams of EtOAc extracts previously lyophilized were subjected to chromatography on a $100 \mathrm{~cm} \times 5 \mathrm{~cm}$ column containing $500 \mathrm{~g}$ of silica gel 60 Merck $(70-230 \mathrm{mesh})$.

The elution was made using a gradient of increasing polarity with $n$-hexane; $n$-hexane- $\mathrm{CHCl}_{3}$ from $1 \%$ to $90 \%$ in $\mathrm{CHCl}_{3} ; \mathrm{CHCl}_{3} ; \mathrm{CHCl}_{3}{ }^{-}$ethanol from $1 \%$ to $75 \% \mathrm{~m}$ ethanol; ethanol (98\%). Solvent in each fraction was evaporated until dryness under vacuum and the extract was lyophilized to yield 14 and 13 fractions for $P$. carolinensis and $P$. rosea respectively. In general $100 \mathrm{~mL}$ of fraction was collected except for eluted from $\mathrm{CHCl}_{3}$ - ethanol from $1 \%$ to $2 \%$ in ethanol where $75 \mathrm{~mL}$ were collected.

One gram of the $n$-BuOH leaf crude extract of both species was separately subjected to the chromatography column using $100 \mathrm{~g}$ of Sephadex LH-20, Pharmacia. Successive elutions through a conventional column $(80 \mathrm{~cm}$ $\times 2 \mathrm{~cm}$ ) with methanol were performed, and $20 \mathrm{~mL}$ fractions were collected. Solvent in each fraction was evaporated until dryness under vacuum and extract was lyophilized to yield six and seven fractions for $P$. carolinensis and $P$. rosea respectively. Silica gel 60 F254 Thin layer chromatography plates were used for determining the end of the fraction collected. They were visualized under 254 and $365 \mathrm{~nm}$ ultraviolet light and subsequently sprayed with a solution of cerium sulfate (IV) in sulfuric acid (65\%).

\section{Antioxidant potentials and total phenolic compound measurement}

The amount of total phenolic compounds of the different crude extracts was determined using Folin-Ciocalteu's reagent and gallic acid as standard. The absorbance at $765 \mathrm{~nm}$ was recorded. ${ }^{20}$ Results of phenolic determination were expressed in milligrams of gallic acid equivalents per gram of dry extract (mg GAE/gDE). The radical scavenging capacity was examined by the reduction of DPPH free radical in methanol. ${ }^{21,22}$ The ORAC assay was also assayed. ${ }^{23}$ Results of the antioxidant potential of fractions were expressed in milligrams of Trolox equivalents per gram of dry extract (mg TE/g DE), whereas antioxidant potential of pure compounds was expressed in $\mu \mathrm{mol} \mathrm{TE} / \mathrm{mmol}$. Each sample was analyzed in triplicates. 


\section{Flavonoid analyses}

HPLC analyses were conducted with a Merck Hitachi La Chrom Elite liquid chromatography equipped with an L-2455 photodiode array detector. $10 \mu \mathrm{L}$ of each sample was injected in an analytical Grace Davison Grace Smart RP C-18 column $(250 \mathrm{~mm} \times 4.6 \mathrm{~mm}, 5 \mu \mathrm{m})$ at $30^{\circ} \mathrm{C}$.

Mobile phase A consisted in $0.05 \%$ trifluoracetic acid (TFA) and mobile phase B was acetonitrile $\left(\mathrm{CH}_{3} \mathrm{CN}\right)$. The gradient elution was performed from $0 \% \mathrm{~B}$ to $65 \% \mathrm{~B}$ in $40 \mathrm{~min}$ at flow rate of $1 \mathrm{~mL} / \mathrm{min}$. Results were expressed in milligrams per gram of dry weight.

\section{Phenolic acids}

The mobile phase for chromatographic separation consisted of the solvent A (acetic acid 2\%) and solvent B $\left(\mathrm{CH}_{3} \mathrm{CN}\right)$ in the gradient. The flow rate was $0.5 \mathrm{~mL} / \mathrm{min}$ and the gradient was the following: Until $32 \mathrm{~min}(95 \%$ A, 5\% B); 35-40 min (100\% A, 0\% B); $63 \min (70 \%$ A, 30\% B), $73 \min (0 \%$ A, $100 \%$ B).

\section{Caffeoylquinic acid (CQA) derivatives}

Mobile phase A consisted in TFA (0.05\%) and mobile phase $\mathrm{B}$ was $\mathrm{CH}_{3} \mathrm{CN}$. The flow rate was $1.0 \mathrm{~mL} / \mathrm{min}$ and the gradient was the following: 0-5 $\min (100 \% \mathrm{~A})$; 5-45 $\mathrm{min}(35 \% \mathrm{~A}, 65 \% \mathrm{~B})$. The absorption wavelength was monitored at $328 \mathrm{~nm}$. Six CQA derivatives: 3-caffeoylquinic acid (3-CQA), 3,4-dicaffeoylquinic acid, 3,5dicaffeoylquinic acid, 4,5-dicaffeoylquinic acid, 3,4,5-tricaffeoylquinic acid, 1,3,4,5-tetracaffeoylquinic acid (tetra-CQA) were quantified. 3-CQA was purchased from Sigma and di and tri-CQA derivatives from Biopurity Phytochemicals Limited, while tetra-CQA was purified previously by HPLC. ${ }^{24}$

\section{Statistical analyses}

Values were expressed as means of three replicates determinations \pm standard deviation. Variance analysis, using Turkey HSD's post-test $P<0.05$, was applied.

\section{RESULTS}

\section{Evaluation of the antioxidant potential of fractions from polar extracts}

The in vitro antioxidant potential and the estimation of phenolic content of several fractions obtained by fractionation from EtOAc and $n-\mathrm{BuOH}$ leaf crude extracts of $P$. carolinensis and $P$. rosea were measured, and they are shown in Table 1.

In general, the estimation of phenolic content was considerably higher in fractions from both $n$-BuOH extracts than those obtained from EtOAc ones. In EtOAc extracts of $P$. carolinensis, the highest phenolic contents were found in the fractions (11-14) varying from $5.6 \pm 0.2$ to $21.0 \pm 0.2 \mathrm{mg}$ GAE/gDE. In $P$. rosea, phenolics were only detected in the most polar fractions (10-13) from $1.1 \pm 0.1$ to $4.0 \pm 0.2 \mathrm{mg} \mathrm{GAE} / \mathrm{g}$ DE. In both extracts, the highest contents were found in the most of polar fractions eluted (fraction 14 of P. carolinensis and fraction 13 for $P$. rosea).

Phenolic content in $n$-BuOH extracts from $P$. carolinensis varied from $13.8 \pm 0.2$ to $159.5 \pm 3.7 \mathrm{mg}$ GAE/g DE in different fractions, with the highest content in fractions 2, 4 and 6 and in $P$. rosea from $2.6 \pm 0.2$ to $180 \pm 10.5$ $\mathrm{mg} \mathrm{GAE} / \mathrm{g}$ DE with the highest contents in fraction 3, 5, 6 and 7.

DPPH scavenging activity of the different fractions showed a similar trend to those obtained from phenolic compound assays. Fractions eluted from $n$ - $\mathrm{BuOH}$ extracts showed in general, considerably higher antioxidant potential than those obtained from EtOAc extracts. Generally, the fractions with the highest concentrations in phenolic compounds were also those with the highest antioxidant potential. In EtOAc extracts, the highest level of antioxidants was also detected in the most polar fractions in the range of $15.8 \pm 0.5-26.5 \pm 0.7 \mathrm{mg} \mathrm{TE} / \mathrm{g} \mathrm{DE}$ for $P$. carolinensis and for $P$. rosea.

The ORAC assay revealed the highest antioxidant potential in fraction 11-14 and 8-13 from EtOAc extracts of $P$. carolinensis and $P$. rosea, respectively. The highest antioxidant potential from $n$-BuOH extracts were measured in fractions 2, 4 and 6 for P. carolinensis and in fractions 3, 6 and 7 for P. rosea, the same fractions as revealed with DPPH. 


\section{Phenolic profile in polar fractions of $P$. carolinensis and $P$. rosea}

The fractions from EtOAc and $n-\mathrm{BuOH}$ extracts of the species P. carolinensis and P. rosea were analyzed by reversed phase-HPLC and results were expressed in mg of phenolic/ gDW (Table 2).

\section{Antioxidant potential of pure phenolic compounds}

As an attempt to correlate the antioxidant potential to specific flavonoids and phenolic acids detected in Cuban Pluchea species we evaluated the DPPH and ORAC antioxidant potential of each compounds and compared with the standard Trolox (Figure 1).

The majority of the flavonoids showed lower values of DPPH antioxidant potential than Trolox. The group of isoflavones, apigenin and casticin (the most methoxylated flavonols) did not react with the DPPH. On the other hand, luteolin, herbacetin, hyperoside and quercetagetin showed the highest values of antioxidant potential (Figure 1a). Ferulic acid, caffeic acid and rosmarinic acid displayed lower antioxidant potential than Trolox (Figure 1b). Tri and tetra-CQA derivatives showed the best antioxidant potential. All the phenolics assayed, exhibited a higher ORAC antioxidant activity than Trolox. The biggest differences were observed for the flavonols herbacetin, quercitrin and spiraeoside that showed an antioxidant potential about 8 times higher than Trolox (Figure 1a and b). The DPPH and ORAC antioxidant potential of phenolic compounds showed very low correlation coefficients.

Table 1: Antioxidant capacity (DPPH and ORAC) and total phenolic content of fractions from $5 \mathrm{~g}$ of EtOAc and $1 \mathrm{~g}$ of $n-\mathrm{BuOH}$ leaf extracts of $P$. carolinensis and $P$. rosea

\begin{tabular}{|c|c|c|c|c|c|c|}
\hline \multirow[t]{2}{*}{ Fraction } & $\begin{array}{c}\text { Phenolics } \\
\text { (mg GAE/gDE) }\end{array}$ & $\begin{array}{c}\text { DPPH } \\
(\mathrm{mgTE} / \mathrm{gDE}) \\
\end{array}$ & $\begin{array}{c}\text { ORAC } \\
(\mathrm{mgTE} / \mathrm{gDE}) \\
\end{array}$ & $\begin{array}{c}\text { Phenolics } \\
\text { (mgGAE/gDE) }\end{array}$ & $\begin{array}{c}\text { DPPH } \\
\text { (mgTE/gDE) } \\
\end{array}$ & $\begin{array}{c}\text { ORAC } \\
\text { (mgTE/gDE) }\end{array}$ \\
\hline & \multicolumn{3}{|c|}{ P. carolinensis $($ EtOAc) } & \multicolumn{3}{|c|}{ P. rosea $($ EtOAc) } \\
\hline 1 & $\mathrm{t}$ & $11.3 \pm 3.6^{\mathrm{d}}$ & $7.0 \pm 1.1^{\mathrm{g}}$ & - & - & - \\
\hline 2 & $\mathrm{t}$ & $\mathrm{t}$ & $10.2 \pm 2.6^{\mathrm{f}}$ & - & - & - \\
\hline 3 & $\mathrm{t}$ & $\mathrm{t}$ & $14.0 \pm 0.5^{\mathrm{f}}$ & - & - & - \\
\hline 4 & $\mathrm{t}$ & $\mathrm{t}$ & $13.8 \pm 0.1^{\mathrm{f}}$ & - & - & - \\
\hline 5 & $1.1 \pm 0.1^{\mathrm{f}}$ & $10.5 \pm 0.1^{\mathrm{d}}$ & $4.3 \pm 0.5^{\mathrm{h}}$ & - & - & - \\
\hline 6 & $\mathrm{t}$ & $\mathrm{t}$ & $\mathrm{t}$ & - & - & - \\
\hline 7 & $\mathrm{t}$ & $\mathrm{t}$ & $2.7 \pm 0.8$ & - & - & - \\
\hline 8 & $1.6 \pm 0.1^{\mathrm{f}}$ & $2.0 \pm 0.1^{\mathrm{f}}$ & $5.2 \pm 1.2^{\mathrm{h}}$ & - & - & $16.1 \pm 0.9^{c}$ \\
\hline 9 & $2.1 \pm 0.1^{\mathrm{e}}$ & $2.1 \pm 0.1^{\mathrm{f}}$ & $23.9 \pm 1.7^{\mathrm{e}}$ & - & - & $1.2 \pm 0.1^{\mathrm{e}}$ \\
\hline 10 & $1.6 \pm 0.1^{\mathrm{f}}$ & $2.0 \pm 0.2^{\mathrm{f}}$ & $12.5 \pm 1.5^{\mathrm{f}}$ & $2.4 \pm 0.1^{\mathrm{c}}$ & $2.7 \pm 0.2^{c}$ & $13.5 \pm 2.0^{c}$ \\
\hline 11 & $5.6 \pm 0.2^{\mathrm{d}}$ & $7.4 \pm 0.4$ & $50.0 \pm 0.6^{c}$ & $3.6 \pm 0.2^{b}$ & $6.4 \pm 0.6^{b}$ & $21.2 \pm 0.9^{b}$ \\
\hline 12 & $10.3 \pm 0.2^{c}$ & $15.8 \pm 0.5^{\mathrm{c}}$ & $36.5 \pm 1.2^{\mathrm{d}}$ & $1.1 \pm 0.1^{\mathrm{d}}$ & $1.7 \pm 0.1^{\mathrm{d}}$ & $8.0 \pm 0.3^{\mathrm{d}}$ \\
\hline 13 & $13.4 \pm 0.3^{\mathrm{b}}$ & $20.7 \pm 0.1^{\mathrm{b}}$ & $90.4 \pm 9.4^{\mathrm{b}}$ & $4.0 \pm 0.2^{\mathrm{a}}$ & $8.7 \pm 0.4^{\mathrm{a}}$ & $57.0 \pm 2.6^{\mathrm{a}}$ \\
\hline 14 & $21.0 \pm 0.2^{\mathrm{a}}$ & $26.5 \pm 0.7^{\mathrm{a}}$ & $107.1 \pm 5.2^{\mathrm{a}}$ & $\mathrm{Nf}$ & $\mathrm{Nf}$ & $\mathrm{Nf}$ \\
\hline Fraction & \multicolumn{3}{|c|}{$P$. carolinensis $(n-\mathrm{BuOH})$} & \multicolumn{3}{|c|}{ P. rosea $(n-\mathrm{BuOH})$} \\
\hline 1 & $\mathrm{t}$ & $\mathrm{t}$ & $\mathrm{t}$ & $2.6 \pm 0.2^{\mathrm{f}}$ & $3.7 \pm 0.1^{\mathrm{f}}$ & $19.8 \pm 0.6^{\mathrm{e}}$ \\
\hline 2 & $125.4 \pm 2.8^{\mathrm{c}}$ & $192.1 \pm 0.3^{\mathrm{a}}$ & $543.0 \pm 64.6^{\mathrm{a}}$ & $24.5 \pm 0.9^{\mathrm{e}}$ & $38 \pm 0.2^{\mathrm{e}}$ & $171.3 \pm 11.6^{\mathrm{c}}$ \\
\hline 3 & $29.1 \pm 1.3^{\mathrm{d}}$ & $54.8 \pm 0.3^{\mathrm{c}}$ & $121.2 \pm 7.1^{\mathrm{c}}$ & $98.4 \pm 2.1^{\mathrm{c}}$ & $156.3 \pm 0.1^{\mathrm{b}}$ & $401.3 \pm 16.1^{\mathrm{a}}$ \\
\hline 4 & $150.1 \pm 1.4^{\mathrm{b}}$ & $162.4 \pm 0.6^{\mathrm{b}}$ & $501.4 \pm 49.7^{\mathrm{a}}$ & $70.2 \pm 1.8^{\mathrm{d}}$ & $91.7 \pm 0.3^{\mathrm{c}}$ & $120.9 \pm 13.8^{d}$ \\
\hline 5 & $13.8 \pm 0.2^{\mathrm{e}}$ & $26.5 \pm 0.1^{\mathrm{d}}$ & $36.4 \pm 1.1^{\mathrm{d}}$ & $89.8 \pm 6.2^{c}$ & $82.3 \pm 0.3^{\mathrm{d}}$ & $162.9 \pm 14.7^{\mathrm{C}}$ \\
\hline 6 & $159.5 \pm 3.7^{\mathrm{a}}$ & $181.0 \pm 0.1^{\mathrm{a}}$ & $383.1 \pm 20.6^{b}$ & $109.1 \pm 5.2^{\mathrm{b}}$ & $156.7 \pm 0.2^{\mathrm{b}}$ & $415.4 \pm 15.3^{\mathrm{a}}$ \\
\hline 7 & $\mathrm{Nf}$ & $\mathrm{Nf}$ & $\mathrm{Nf}$ & $180 \pm 10.5^{\mathrm{a}}$ & $188.1 \pm 5.5^{\mathrm{a}}$ & $336.3 \pm 45.9^{b}$ \\
\hline
\end{tabular}

Nf: No fraction was eluted, -: No detection, $\mathrm{t}: \leq 1 \mathrm{mg}$ TE or GAE/gDE (dry extract). The antioxidant capacity for each individual technique was compared between fractions of the same species. Different letters indicated significant statistical differences. Coefficients of variance for total phenolics, DPPH and ORAC were lower than 10\%. DPPH: 2,2-diphenyl-1 picrylhydrazyl, ORAC: Oxygen radical scavenging capacity, mg GAE/g DE: Milligrams of gallic acid equivalents per gram of dry extract, mg TE/g DE: Milligrams of Trolox equivalents per gram of dry extract, EtOAc: Ethyl acetate, $n$-BuOH: $n$-butanol, $P$. carolinensis: Pluchea carolinensis, $P$. rosea: Pluchea rosea 
Table 2: Quantification of phenolic compounds in P. carolinensis and P. rosea by HPLC-diode array detector

\begin{tabular}{lccc}
\hline Classification & Phenolics & P. carolinensis & P. rosea \\
\hline Cinnamic & Caffeic acid & $0.29 \pm 0.03^{\mathrm{h}}$ & $0.28 \pm 0.01^{\mathrm{f}}$ \\
acids & Ferulic acid & $* 0.11 \pm 0.01^{\mathrm{k}}$ & $\mathrm{Nd}$ \\
& Rosmarinic acid & $0.69 \pm 0.02^{\mathrm{f}}$ & $\mathrm{Nd}$ \\
Caffeoylquinic & 3-CQA & $1.49 \pm 0.21^{\mathrm{e}}$ & $0.30 \pm 0.01^{\mathrm{f}}$ \\
acid & 3,4-diCQA & $8.99 \pm 0.30^{\mathrm{b}}$ & $1.20 \pm 0.05^{\mathrm{e}}$ \\
derivatives & 3,5-diCQA & $3.84 \pm 0.31^{\mathrm{c}}$ & $3.95 \pm 0.09^{\mathrm{d}}$ \\
& 4,5-diCQA & $21.2 \pm 0.4^{\mathrm{a}}$ & $25.6 \pm 0.2^{\mathrm{a}}$ \\
& triCQA & $2.42 \pm 0.09^{\mathrm{d}}$ & $8.92 \pm 0.32^{\mathrm{b}}$ \\
& tetra-CQA & $1.44 \pm 0.14^{\mathrm{e}}$ & $5.79 \pm 0.22^{\mathrm{c}}$ \\
Flavonoids & Casticin & $\mathrm{Nd}$ & $* 0.19 \pm 0.01^{\mathrm{g}}$ \\
& Herbacetin & $0.01 \pm 0^{\mathrm{j}}$ & $\mathrm{Nd}$ \\
& Isorhamnetin & $0.13 \pm 0.04^{\mathrm{i}}$ & $\mathrm{Nd}$ \\
& Kaempferol & $0.11 \pm 0.04^{\mathrm{i}}$ & $\mathrm{Nd}$ \\
& Luteolin & $0.11 \pm 0.01^{\mathrm{i}}$ & $\mathrm{Nd}$ \\
& Myricetin & $0.43 \pm 0.03^{\mathrm{g}}$ & $\mathrm{Nd}$ \\
& Quercetin & $0.12 \pm 0.01^{\mathrm{i}}$ & $\mathrm{Nd}$ \\
& Quercitrin & $0.06 \pm 0.01^{\mathrm{j}}$ & $\mathrm{Nd}$ \\
& Quercetagetin & $0.02 \pm 0^{\mathrm{j}}$ & $* 0.24 \pm 0.04^{\mathrm{g}}$ \\
& Rutin & $\mathrm{Nd}$ & $0.4 \pm 0.02^{\mathrm{f}}$
\end{tabular}

Nd: Not detected, 3-CQA: 3-caffeoylquinic acid, 3,4-diCQA: 3,4-dicaffeoylquinic acid, 3,5-diCQA: 3,5-dicaffeoylquinic acid, 4,5-diCQA: 4,5-dicaffeoylquinic acid triCQA: 3,4,5-tricaffeoylquinic acid, tetra-CQA: 1,3,4,5-tetracaffeoylquinic acid. In each column, different letters mean significant difference at $P \leq 0.05$. Results are expressed as milligrams of phenolic per gram of dry weight. $*\left(10^{-2}\right)$. DAD: Diode array detector HPLC: High performance liquid chromatography

In our study, several phenolic compounds were identified, therefore, we decided to estimate the contribution of the total phenolic content as well as of each individual pure chemical quantified to the total antioxidant potential of the crude extracts. The sum of DPPH and ORAC antioxidant potential of each individual phenolic identified by HPLC was computed. The contributions to the total antioxidant potential of the crude extract are shown in Table 3.

The estimation to the contribution of the antioxidant potential of the phytochemicals identified to the total antioxidant potential (DPPH and ORAQ of the leaf extracts from P. carolinensis and P. rosea (without forgetting we are not taking into account the intermolecular interactions as well, only the sum of the antioxidant potential of each individual phenolic) are shown in Figure 2.

The contributions of all the phytochemicals (expressed in percent) to the DPPH antioxidant potential in the leaf crude extracts of $P$. carolinensis was $38.7 \%$ and in $P$. rosea $49.7 \%$. Additionally, the ORAC contribution in $P$. carolinensis was $53.2 \%$ and in $P$. rosea $52.4 \%$. The phenolic compounds identified and quantified in both species contribute significantly to the total antioxidant potential of the crude extracts of both species. Three phytochemical groups were mainly identified in both species: Cinnamic acids, CQAs and flavonoids (flavones and flavonols). The contribution of each groups to the total antioxidant potential of the leaf extracts of $P$. carolinensis'were calculated independently (Figure 3).

\section{DISCUSSION}

The highest antioxidant potentials and phenolic content were previously reported in polar EtOAc and $n$-BuOH leaf crude extracts of the species $P$. carolinensis and $P$. rosea. ${ }^{10}$ As a first step for identifying the major antioxidant constituents, the EtOAc and «-BuOH extracts of both species were fractionated. We correlated the antioxidant potential and the phenolic content in $n-\mathrm{BuOH}$ fractions of both species. The highest correlation coefficients were observed in $P$. carolinensis and $P$. rosea for DPPH versus, total phenolics with $r^{2}=0.9365$ and $r^{2}=0.8793$ respectively. Correlation coefficients of ORAC vs. phenolic were lower with $r^{2}=0.8715$ and $r^{2}=$ 0.511 for $P$. carolinensis and $P$. rosea respectively. 
Figure 1: Antioxidant capacity of phenolic pure compounds: a (flavonoids) and b (phenolic acids). Results for DPPH and ORAC were expressed in $\mu \mathrm{mol} T E / m m o l$. All the analyses were performed in triplicate.

Abbreviations: Apigenin (A), Luteolin (L), Daidzein (Dze), Daidzin (Dzi), Genistein (Gte), Genisttn (Gti), Ononin (O), Casticin (C), Herbacetin (H), Hyperoside (Hy), Isorhamnetin (I), Quercetagettn (Qtg), Quercitrin $(Q t)$, Spiraeoside $(S)$, Ferulic acid $(F$ a), Rosmarinic acid $(R$ a), caffeic acid $(C$ a), 3 -caffeoylquinic acid or chlorogenic acid (3-CQA), 3,4-dicaffeoylquinic acid (3,4-CQA), 3,5-dicafeoylquinic acid (3,5-CQA), 4,5dicaffeoylquinic acid (4,5-CQA), 3,4,5-tricaffeoylquinic acid (triCQA), 1,3,4,5-tetracaffeoylquinic acid (tetraCQA), Trolox $(T)$, standard (STD). * indicate positive significant statistical differences compared to the standard. Tukey HSD's post hoc test $P<0.05, n=3$
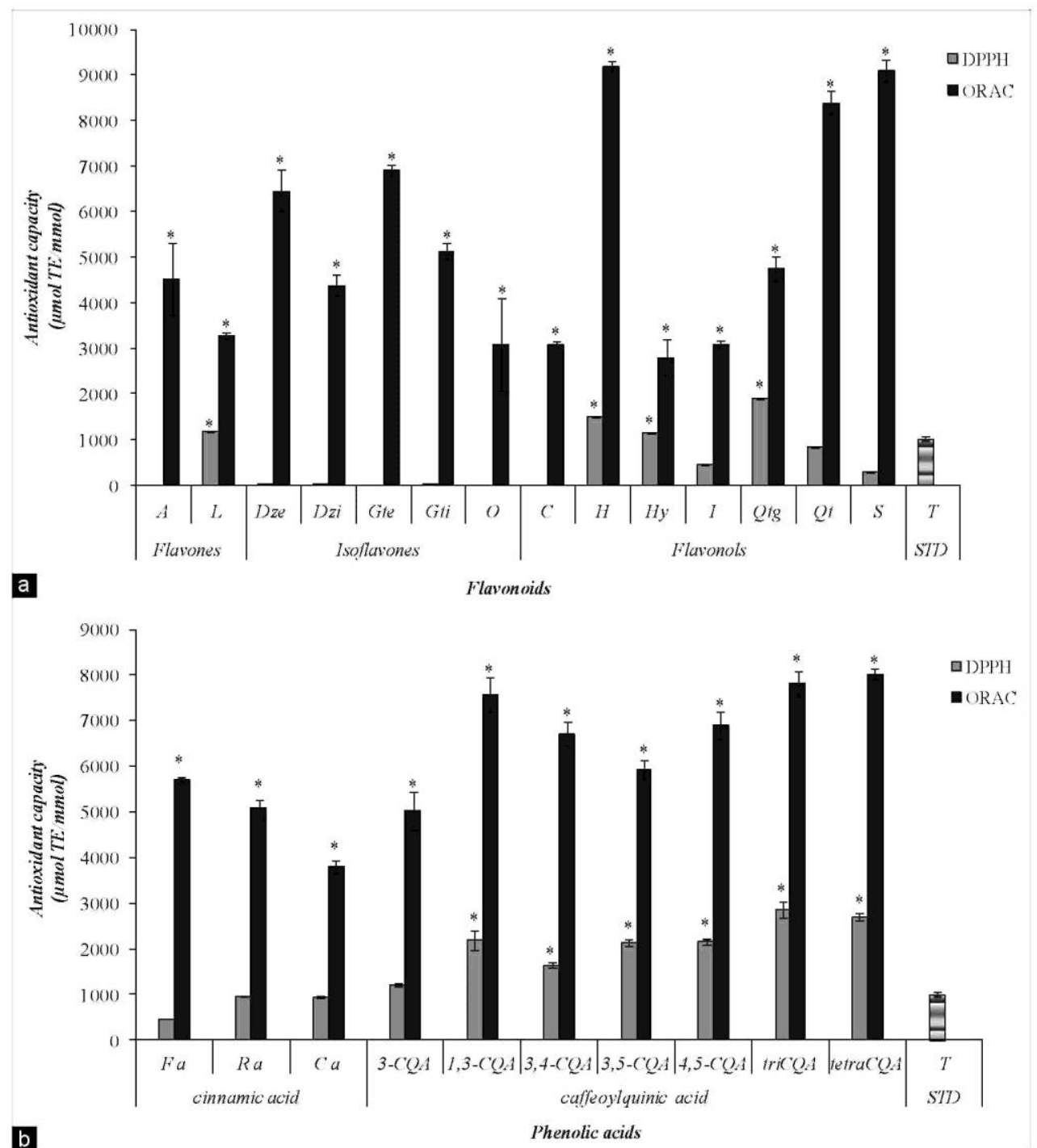
Figure 2: Estimation of the contribution of the antioxidant potential of all the phenolics to the total antioxidant potentials (2,2-diphenyl-1 picrylhydrazyl and oxygen radical scavenging capacity) of the leaf extracts of Pluchea carolinensis and Pluchea rosea

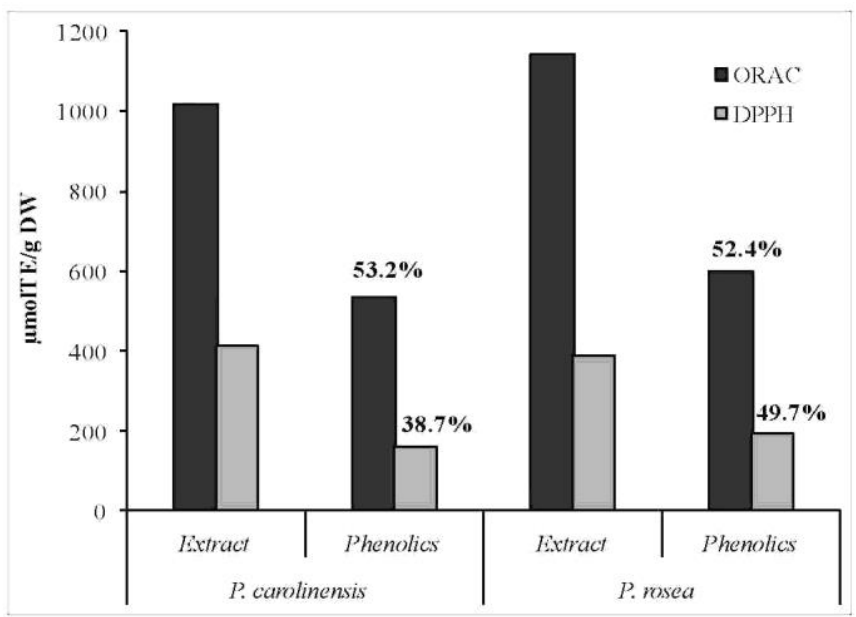

Figure 3: Contribution of each phytochemical group to the 2,2-diphenyl-1 picrylhydrazyl and oxygen radical scavenging capacity antioxidant potential of the leaf extracts of Pluchea carolinensis

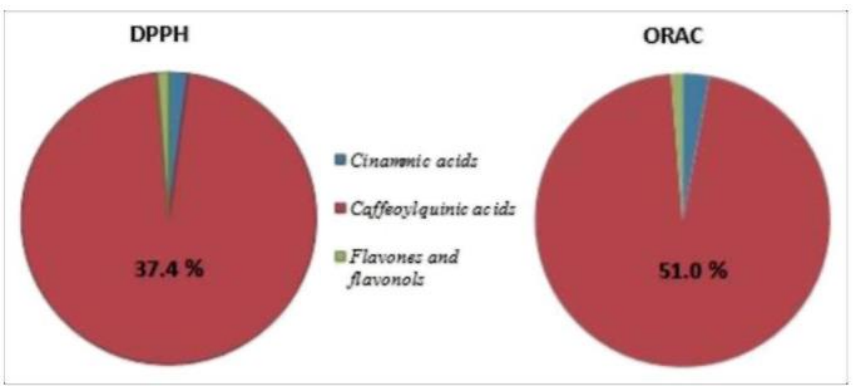

Table 3: Determination of the contribution to the antioxidant capacity (2,2-diphenyl-l picrylhydrazyl and oxygen radical scavenging capacity) of each pure compound identified in Pluchea carolinensis

\begin{tabular}{lcccc}
\hline Chemicals & $\begin{array}{c}\text { Phenolics } \\
\text { identified }\end{array}$ & $\begin{array}{c}\text { Concentration } \\
(\mathbf{m g} / \mathbf{g D W})\end{array}$ & $\begin{array}{c}\boldsymbol{\mu} \mathbf{m o l} \\
\mathbf{D P P H}\end{array}$ & $\begin{array}{c}\text { TE/g DW } \\
\text { ORAC }\end{array}$ \\
\hline Cinnamic acid & Caffeic acid & 0.29 & 1.53 & 6.16 \\
& Ferulic acid & 0.0011 & 0 & 0.03 \\
RQAs & Rosmarinic acid & 0.69 & 1.84 & 9.72 \\
& 3-CQA & 1.49 & 5.08 & 27.19 \\
& 3,4-diCQA & 8.99 & 28.44 & 116.59 \\
& 3,5-diCQA & 3.84 & 15.80 & 43.91 \\
& 4,5-diCQA & 21.17 & 88.69 & 282.38 \\
Flavonoids & triCQA & 2.42 & 10.17 & 27.88 \\
& tetra-CQA & 1.44 & 6.22 & 20.13 \\
& Herbacetin & 0.01 & 0.04 & 0.24 \\
& Isorhamnetin & 0.13 & 0.19 & 1.27 \\
& Kaempferol & 0.11 & 0.10 & 0.72 \\
& Luteolin & 0.11 & 0.45 & 1.26 \\
& Myricetin & 0.44 & 0.8 & 1.55 \\
& Quercetin & 0.12 & 0.14 & 0.42 \\
Phytochemicals & Quercetagetin & 0.02 & 0.12 & 0.3 \\
Crude extract & & 0.06 & 0.11 & 1.12 \\
\hline
\end{tabular}

Values of the antioxidant capacity for quercetin, kaempferol, myricetin previously determined by Tabart et al. (2009) were used to estimate the contribution of these molecules to the total antioxidant of crude extracts. CQAs: Caffeoylquinic acids, DPPH: 2,2-diphenyl-1 picrylhydrazyl, ORAC: oxygen radical scavenging capacity, 3-CQA: 3-caffeoylquinic acid, 3,4-diCQA: 3,4-dicaffeoylquinic acid 3,5- 
Various analytical methods were developed to identify and quantify some abundant phenolic compounds in plant kingdom as well as others previously describe in the genus Pluchea. ${ }^{16,25,26}$ Nineteen phenolics were identified in the fractions: Three cinnamic acids, six CQAs, a flavone and nine flavonols including seven aglycone and two glycoside forms. In general, the CQAs were detected in higher concentrations than the other phenolic acids and flavonoids measured. The 3,4-CQA isomer was the compound prevalent in both species. Seven of the flavonoids identified were navonol aglycones, two navonol glycosides (quercitrin and rutin) and one flavone aglycone (luteolin). These results are consistent with those observed in the genus Pluchea in which the occurrence of flavonoid aglycones is more frequently reported than flavonoid glycosides. ${ }^{15-17,25,26}$ Some of the phytochemicals were identified for the first time in $P$. carolinensis and in $P$. rosea and the antioxidant potential was assayed. These results are in accordance with those observed for flavonoids and phenolic acids. ${ }^{27}$

The antioxidant potential of plant organs from Cuban species of Pluchea has been recently assayed. Leaves followed by inflorescences were the plant organs with highest in vitro antioxidant potential. ${ }^{28}$ It's well-known that a crude extract is composed by a mixture of several phytochemicals, sometimes with insufficient structural knowledge. Moreover, intermolecular interactions trigger antagonistic or synergistic effects in the antioxidant properties of natural mixtures. The understanding of this phenomenon is not very clear until today thus it is difficult to predict the behavior of a mixture of various chemicals. Hence, the contribution to the antioxidant potential of each pure chemical to the total antioxidant potential of the crude extracts is very difficult to establish. Thus, results showed that CQAs contribute more significantly to the total antioxidant potentials of the crude extract. Cinnamic acids and flavonoids contributed very slightly. This fact suggests that the antioxidant potential found in P. carolinensis is due, at least partially, to the CQAs.

\section{CONCLUSION}

The DPPH and ORAC antioxidant potential of several fractions from polar EtOAc and $n$-BuOH extracts of the species $P$. carolinensisand $P$. rosea were evaluated. Frequently, fractions from $n$-BuOH extracts showed higher antioxidant potential than those from EtOAc extracts. In general, highest correlation between antioxidant potential and phenolic content by Folin-Ciocalteu assay were computed. Various antioxidant compounds were identified in leaf extracts, almost all the phytochemicals were reported for the first time in Cuban Pluchea species. Additionally, rosmarinic acid, ferulic acid, quercetagetin, herbacetin and quercitrin were identified for the first time in Pluchea genus. The phenolic identified contributes significantly to the total antioxidant potential of the crude extracts (around 50\%). Apparently, CQA derivatives are the metabolites which contribute more significantly to the total antioxidant potential of the crude extracts.

\section{REFERENCES}

1. Bremer K. Asteraceae: Cladistics \& Classification. Portland, Oregon: Timber Press; 1994. p. 30-40.

2. Fernández F, Torres M. Evaluation of Pluchea carolinensis extracts as antioxidants by the epinephrine oxidation method. Fitoterapia 2006;77:221-6

3. Marwah RG, Fatope MO, AI Mahrooqi R, Varma GB, Al Abadi H, Al-Burtamani SK. Antioxidant capacity of some edible and wound healing plants in Oman. Food Chem 2007;101:465-70.

4. Abraham A, Hernández N, Misas CA. Potential antineoplastic activity of Cuban plants. Rev Cubana Farm 1981;15:71-7.

5. Laurent D, Vilaseca LA, Chantraine JM, Ballivian C, Saavedra G, Ibañez R. Insecticidal activity of essential oils on Triatoma infestans. Phytother Res 1997;11:285-90.

6. Rosales VP, Gross MC, Rosales R, Garcia RC, León JE, Vidal M. Evaluación farmacológica de Pluchea carolinensis Jacq. (salvia de playa) en animales de experimentación. Rev Cubana Plant Med 1999;3:65-7.

7. Inderjit SK, Dakshini KM. Allelopathic potential of the phenolics from the roots of Pluchea lanceolata. Physiol Plant 1994;92:571-6.

8. Nagmoti DM, Khatri DK, Juvekar PR, Juvenkar AR. Antioxidant activity free radical-scavenging potential of Pithecellobium dulce Benth seed extracts. Free Rad Antioxid 2012;2:37-43.

9. PereraWH,Tabart J,Sipel A,Gómez A, Payo AL, Kevers C,et al. Antioxidant capacity of three Cuban species of the genus Pluchea Cass. 
Published in : Free Radicals and Antioxidants (2014), vol. 4, iss. 2.

Status : Postprint (Author's version)

(Asteraceae). J Food Biochem 2010;34:249-61.

10. Gonçalves AF, Friedrich RB, Boligon AA, Piana M, Beck RC, Athayde ML. Antioxidant capacity, total phenolic contents and HPLC determination of rutin in Viola tricolor (L) flowers. Free Rad Antioxid 2012;2:32-7.

11. Pino J, Perera W, Sarduy R, Oviedo R, Quijano C. Essential oil from the stems, leaves and flowers of Pluchea rosea Godfrey and Pluchea purpurascens (Sw.) DC. J Essent Oil Res 2008;20:497-501.

12. Simionatto EL, Júnior AW. Essential Oil of Pluchea quitoc Dc. (Asteraceae). J Essent Oil Res 2007;19:494-7.

13. Ahmed AA, El-Seed HR, Mahmoud AA, El-Douski AA, Zeid IF, Bohlin L. Eudesmane derivatives from Laggera crispata and Pluchea carolinensis. Phytochemistry 1998;49:2421-4.

14. Mahmoud AA. 7-Epi-eudesmanes, eudesmanoic acids, eudesmanolides and other sesquiterpenes from Pluchea dioscoridis. Phytochemistry 1997;45:1633-8.

15. Napralert ${ }^{\mathrm{TM}}$. Data Base of College of Pharmacy of the University of Illinois at Chicago, E.U.; 1975-2009.

16. Wollenweber E, Mann K. Flavonoids and terpenoids from the leaf resin of Pluchea odorata. Z Naturforsch 1985;40:321-4.

17. Chiang MT, Silva M. Anticancer agents from Pluchea chingoyo D.C. Rev Latinoam Quim 1978;9:102-4.

18. Perera WH, González L, PayoAL, Nogueiras C, Delgado G, Oquendo M, et al. Antimicrobial activity of crude extracts and flavonoids from leaves of Pluchea carolinensis (Jacq.) G. Don. Pharmacol Online 2006;3:757-61.

19. Akhila S, Bindu AR, Bindu K, Aleykutty NA. Comparative evaluation of extracts of Citrus limon burm peel for antioxidant activity. J Young Pharm 2009;1:136-40.

20. Determination of substances characteristic of green and black tea-Part 1: Content of total polyphenols in tea - Colorimetric method using Folin-Ciocalteu reagent. ISO/TC 34. ISO 14502-1. Switzerland: International Organization for Standardization; 2006.

21. Lo Scalzo R. Organic acids influence on DPPH scavenging by ascorbic acid. Food Chem 2008;107:40-3.

22. Parasuraman S, Kumar E, Kumar A, Emerson S. Free radical scavenging property and diuretic effect of triglize, a polyherbal formulation in experimental models. J Pharmacol Pharmacother 2010;1:38-41.

23. Wu X, Beecher GR, Holden JM, Haytowitz DB, Gebhardt SE, Prior RL. Lipophilic and hydrophilic antioxidant capacities of common foods in the United States. J Agric Food Chem 2004;52:4026-37.

24. Perera CW. Isolation and identification of antioxidant phytochemicals from Cuban species of the genera Erythroxylum P. Browne and Pluchea Cass. Thesis; 2012. p. 200.

25. Andarwulan N, Batari R, Sandrasari DA, Boiling B, Wijaya N. Flavonoid content and antioxidant activity of vegetables from Indonesia. Food Chem 2010;12:1231-5.

26. Romo de Vivar A, Reyes B, Delgado G, Schlemper EO. Constituents of Pluchea sericea. Structure and stereochemistry of (11S)-11, 13dihydrotessaric acid. Chem Lett 1982;7:957-60

27. Tabart J, Kevers C, Sipel A, Pincemail J, Defraigne JO, Dommes J. Comparative antioxidant capacities of phenolic compounds measured by various tests. Food Chem 2009;113:1226-33.

28. Perera WH, Kevers C, Michiels JA, Spengler Salabarria I, Dommes J. Variation in phenolic constituents and antioxidant capacity of three Cuban species of Pluchea Cass. (Asteraceae) according to plant organs, ex vitro and in vitro growth conditions. J Med Plants Res $2013 ; 7: 2523-32$ 